\title{
Dinaciclib induces immunogenic cell death and enhances anti-PD1-mediated tumor suppression
}

\author{
Dewan Md Sakib Hossain, Sarah Javaid, Mingmei Cai, Chunsheng Zhang, Anandi Sawant, Marlene Hinton, Manjiri Sathe, \\ Jeff Grein, Wendy Blumenschein, Elaine M. Pinheiro, and Alissa Chackerian \\ Merck \& Co. Inc., Kenilworth, New Jersey, USA.
}

\begin{abstract}
Blockade of the checkpoint inhibitor programmed death 1 (PD1) has demonstrated remarkable success in the clinic for the treatment of cancer; however, a majority of tumors are resistant to anti-PD1 monotherapy. Numerous ongoing clinical combination therapy studies will likely reveal additional therapeutics that complement anti-PD1 blockade. Recent studies found that immunogenic cell death (ICD) improves T cell responses against different tumors, thus indicating that ICD may further augment antitumor immunity elicited by anti-PD1. Here, we observed antitumor activity following combinatorial therapy with anti-PD1 Ab and the cyclin-dependent kinase inhibitor dinaciclib in immunocompetent mouse tumor models. Dinaciclib induced a type I IFN gene signature within the tumor, leading us to hypothesize that dinaciclib potentiates the effects of anti-PD1 by eliciting ICD. Indeed, tumor cells treated with dinaciclib showed the hallmarks of ICD including surface calreticulin expression and release of high mobility group box 1 (HMCB1) and ATP. Mice treated with both anti-PD1 and dinaciclib showed increased T cell infiltration and DC activation within the tumor, indicating that this combination improves the overall quality of the immune response generated. These findings identify a potential mechanism for the observed benefit of combining dinaciclib and anti-PD1, in which dinaciclib induces ICD, thereby converting the tumor cell into an endogenous vaccine and boosting the effects of anti-PD1.
\end{abstract}

\section{Introduction}

Anti-programmed death 1 (anti-PD1) mAb treatment has demonstrated efficacy across several different cancer types, and these initial clinical successes have galvanized the field of cancer immunotherapy (1-5). PD1 is expressed by T lymphocytes upon cell activation and on exhausted $\mathrm{T}$ cells that are refractory to stimulation $(5-8)$. PD1 is one of several regulatory molecules that deliver an inhibitory signal to prevent excessive inflammation $(9,10)$. In the context of cancer, blocking the interaction of PD1 with its ligands PD-L1 and/or PD-L2 prevents this immunosuppressive signal and allows tumor-specific $\mathrm{T}$ cells to remain activated and kill tumor cells $(5,6,11-13)$. While some cancer patients treated with anti-PD1 agents have experienced dramatic tumor regressions, the efficacy of anti-PD1 is not universal, and it is evident that the preexisting tumor environment influences the responsiveness to treatment. Positive prognostic factors include the presence of tumor-infiltrating lymphocytes (TILs), PD-L1 expression, high mutational load, expression of neoantigens, and an IFN- $\gamma$ gene signature $(2,14-20)$. Tumors that intrinsically lack antigen presentation or are devoid of $\mathrm{T}$ cells that can respond to antigens are significantly less likely to respond to anti-PD1 (19). Thus, therapies that can create an immunogenic environment within tumors that otherwise are immune suppressed or immunologically barren have the potential to expand the number of patients who could benefit from anti-PD1 treatment.

Conflict of interest: The authors have declared that no conflict of interest exists. Submitted: April 18, 2017; Accepted: November 21, 2017.

Reference information: J Clin Invest. 2018;128(2):644-654.

https://doi.org/10.1172/JCI94586.
One approach to elicit this transformation involves the use of agents that induce immunogenic cell death (ICD) within the tumor. A limited number of cytotoxic agents (e.g., anthracyclines, oxaliplatin, radiation therapy, oncolytic viruses) have been shown to induce ICD (21-28). ICD is characterized by the release or cell-surface expression of highly immunostimulatory damageassociated molecular patterns (DAMPs) by the dying tumor cells. Extracellular release of high mobility group box 1 (HMGB1) and ATP serves to attract and activate antigen-presenting cells (APCs), while the display of calreticulin (CRT) on the dying cell's surface serves as an "eat-me" cue to phagocytes $(25,27,29)$. Additionally, induction of the cancer cell-intrinsic type I IFN pathway has been associated with ICD (30). As a result, the dying tumor cell itself both serves as an endogenous vaccine and attracts immune cells into the tumor microenvironment or draining lymph node. Tumor peptides displayed by professional APCs can activate $\mathrm{T}$ cells, which are now licensed to attack the tumor. This principle is demonstrated in a vaccination scenario, whereby inoculation of mice with tumor cells killed by ICD-inducing agents prevents subsequent growth of live tumor cells $(22,27,28)$. T cells and APCs are involved in mediating the antitumor effects of ICD inducers (31-35). In human breast and colorectal cancer patients treated with anthracyclines or oxaliplatin, favorable clinical outcomes were found to be associated with an increased number of cytotoxic CD8 ${ }^{+} \mathrm{T}$ cells within the tumor $(32,34,35)$. Loss of DC function was found to be a negative predictor of the therapeutic response to anthracyclines or oxaliplatin in both clinical and preclinical settings $(31,33)$. These immunostimulatory properties make ICD-inducing agents attractive candidates for combina- 
A

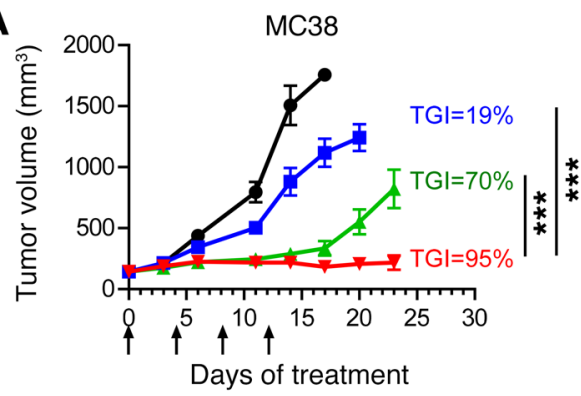

C

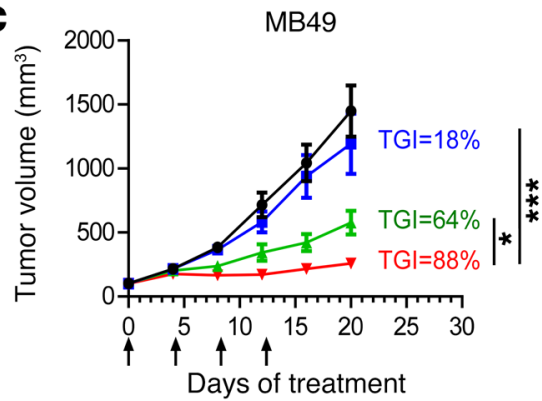

B

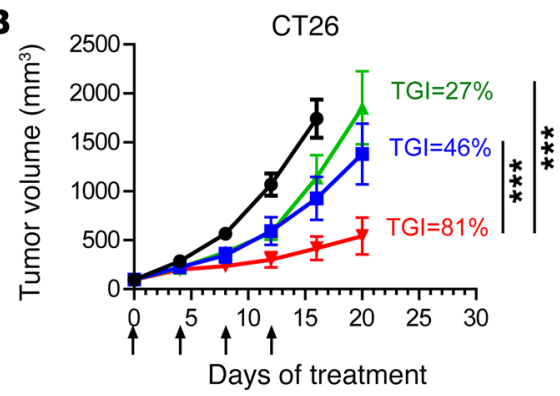

D

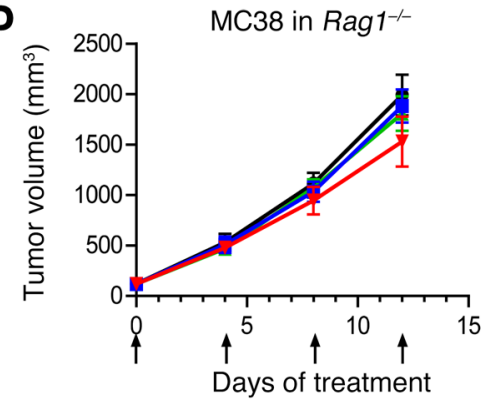

- Isotype + vehicle

- Dinaciclib + isotype

Anti-PD1 + vehicle

Figure 1. Dinaciclib and anti-PD1 combination therapy inhibits tumor growth in syngeneic mouse tumor models. Dinaciclib was tested alone and in combination with antiPD1 mAb in (A and C) C57/BL6], (B) BALB/c, and (D) Rag 1/- $^{-1}$ mice implanted with (A and D) MC38, (B) CT26, or (C) MB49 tumor cells. Tumor volume is represented as the mean \pm SEM. The percentage of TCI on day 20 is presented for each treatment group compared with the control group. Arrows indicate the treatment time points. Data represent at least 2 independent experiments $(n=10-12$ mice/group). ${ }^{* *} P<0.001$ and ${ }^{*} P<0.05$, by 2-way ANOVA with Bonferroni post-test. tion immunotherapy, and initial results in mice suggest that this is a viable path forward (16). However, only a limited number of anticancer drugs have been identified as ICD inducers, and their approved use is restricted to certain cancer types. The efficacy of a particular drug is limited because of the genetic diversity, tissue origin, and local microenvironment of the tumor, thus it is highly desirable to explore whether other anticancer drugs can increase cancer cell immunogenicity and subsequently expand the benefit of anti-PD1 treatment.

Cyclin-dependent kinases (CDKs) are a family of serine/threonine kinases that control cell-cycle progression, and several studies have identified a role for deregulated CDKs in uncontrolled proliferation as well as genomic and chromosomal instability of cancer cells (36). Over the past 20 years, a number of CDK inhibitors have been developed and successfully tested in clinical trials for different tumor types (37). However, a role for CDK inhibitors in inducing ICD has not been described. Here, we demonstrate that the CDK inhibitor dinaciclib (also known as MK-7965 and SCH727965) is capable of eliciting ICD. Dinaciclib is a potent CDK1, -2, -5, and -9 inhibitor that induces apoptosis in different tumor cells and has been shown to be clinically active in refractory chronic lymphocytic leukemia (38-46). These CDK targets regulate the cell cycle (CDK1, -2), control actin polymerization and neuronal function (CDK5), and regulate RNA-polymerase II (CDK9), and their repression can affect $\mathrm{T}$ cell proliferation and migration (47). Thus, our finding that dinaciclib enhances, rather than abrogates, the antitumor efficacy of anti-PD1 Ab in established murine syngeneic tumors was unexpected. Further studies revealed that dinaciclib-treated tumor cells express the hallmarks of ICD (HMGB1, ATP, and CRT), stimulate phagocytic activation of and antigen presentation by DCs, and protect against tumor growth when used in a vaccine setting. Dinaciclib upregulates type IIFN response genes within the tumor as well as PD1 expression on
T cells. When combined with anti-PD1, we saw enhanced recruitment and activation of T cells and APCs in the tumor, resulting in tumor regression. This study suggests that boosting the immunogenicity of the tumor with an ICD inducer such as dinaciclib can augment the overall efficacy of anti-PD1 checkpoint blockade.

\section{Results}

Dinaciclib and anti-PD1 combination therapy inhibits established solid tumor growth in immunocompetent mice. The antitumor effect of dinaciclib and anti-PD1 Ab was tested in 3 murine syngeneic tumor models with varying responsiveness to anti-PD1 monotherapy. Mice with large, established s.c. tumors (MC38 at $150 \mathrm{~mm}^{3}$, CT26 and MB49 at $\sim 100 \mathrm{~mm}^{3}$ ) were treated with dinaciclib and anti-PD1 $\mathrm{Ab}$ alone or in combination every 4 days. In mice, dinaciclib has a 15-minute half-life after a $5-\mathrm{mg} / \mathrm{kg}$ dose (48). To mimic drug exposure in the human clinical setting, in which dinaciclib is given over a 2-hour infusion period, mice were administered 2 doses of dinaciclib $(10 \mathrm{mg} / \mathrm{kg}), 2$ hours apart, on the dosing days. Anti-PD1 mAb was dosed at $5 \mathrm{mg} / \mathrm{kg}$. In all 3 models, dinaciclib plus anti-PD1 combination therapy resulted in more tumor growth inhibition (TGI) than did either treatment alone, with MC38 tumors being the most sensitive to the combination and CT26 tumors showing the biggest differential between combination treatment and monotherapy (Figure 1, A-C). Dinaciclib plus anti-PD1 combination treatment completely eliminated tumors below palpable detection in $25 \%, 10 \%$, and $20 \%$ of mice bearing MC38, CT26, and MB49 tumors, respectively. No complete tumor elimination was observed in the dinaciclib or antiPD1 monotherapy groups. Additionally, no signs of toxicity or weight loss were observed in any of the treatment groups (Supplemental Figure 1; supplemental material available online with this article; https://doi.org/10.1172/JCI94586DS1). The combination benefit of dinaciclib plus anti-PD1 was dependent on the adaptive immune response, as we did not observe TGI in Rag-KO $\left(\mathrm{Rag}^{-/}\right)$mice with 

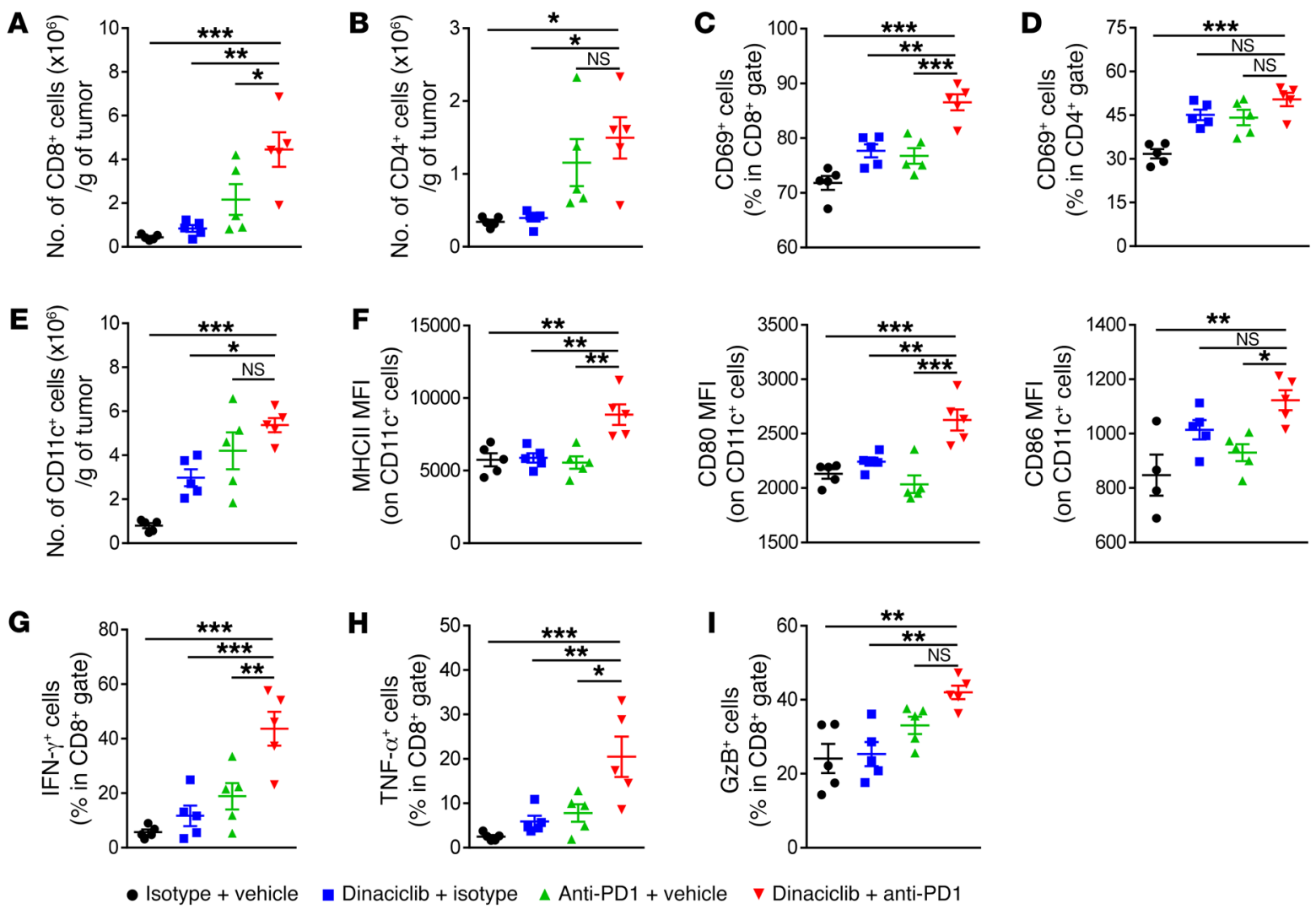

Figure 2. Dinaciclib and anti-PD1 combination therapy induces immune cell infiltration and activation in tumors. Mice with established CT26 tumors were treated with dinaciclib and anti-PD1 mAb as described in Figure 1. Tumors were isolated on day 14, and immune cells were analyzed by flow cytometry ( $n=5$ mice/group). Shown are the numbers of tumor-infiltrating (A) CD8 ${ }^{+} T$ cells, (B) CD4+ $T$ cells, and (E) CD11 $b^{+} C D 11 c^{+} D C s$ in the different treatment groups. Also shown is the activation status of these cell populations as measured by the percentage of CD69+ $\mathrm{CD} 4^{+}$and $\mathrm{CD} 8^{+} \mathrm{T}$ cells (C and $\left.\mathbf{D}\right)$ and $\mathrm{MHCll}$, CD80, and CD86 mean fluorescence intensity (MFI) on DCs (F). For functional analysis, TILs were isolated from dissociated tumors using density-gradient centrifugation. For the detection of intracellular cytokines, harvested TILs were stimulated with PMA and ionomycin in the presence of brefeldin $A$ for 4 hours. Shown are the percentages of (G) IFN- $\gamma^{+},(\mathbf{H})$ TNF- $\alpha^{+}$, and (I) $\mathrm{GzB}^{+} \mathrm{CD}^{+} \mathrm{T}$ cells. Data represent at least 2 independent experiments. ${ }^{* *} P<0.001$, ${ }^{*} P<0.01$, and ${ }^{*} P<0.05$, by 1-way ANOVA with Bonferroni post-test.

established MC38 tumors (Figure 1D). The Rag-KO finding was not surprising, as anti-PD1 activity is T cell dependent. However, it was unexpected that dinaciclib, which has the ability to kill both T cells and tumor cells in vitro, enhanced, rather than abrogated, anti-PD1 activity in the immunocompetent mice.

Treatment with dinaciclib and anti-PD1 increases intratumoral $C D 8^{+} T$ cells and DC activation. To determine whether dinaciclib boosts or inhibits anti-PD1-mediated enhancement of $\mathrm{T}$ cell responses, we examined $\mathrm{T}$ cell infiltration and activation in the tumor. We treated BALB/c mice with established CT26 tumors with dinaciclib and anti-PD1 as before. On day 14 after treatment initiation (i.e., 2 days after the fourth dose), tumors were harvested and analyzed by flow cytometry. Compared with dinaciclib and anti-PD1 monotherapies, we found that combination treatment increased the number of tumor-infiltrating $\mathrm{CD} 8^{+}$and $\mathrm{CD} 4^{+} \mathrm{T}$ cells (Figure 2, A and B), and we observed a similar increase in the number of CD8 ${ }^{+} \mathrm{T}$ cells in the MC38 and MB49 tumor models (Supplemental Figure 2, A and C). Additionally, a higher proportion of tumor-infiltrating $\mathrm{T}$ cells in the treatment groups expressed the $\mathrm{T}$ cell activation marker CD69 compared with the controls, with the highest proportion seen in the combination treatment group (Figure 2, C and D). These effects appeared to be limited to the tumor, as treatment had no impact on $\mathrm{T}$ cell populations in the spleen (Supplemental Figure 3). To address whether combination treatment enhances $\mathrm{T}$ cell function, we performed intracellular cytokine staining on tumor-infiltrating cells isolated from dissociated tumors. Compared with dinaciclib and anti-PD1 monotherapies, combination treatment increased the percentage of IFN- $\gamma$ expression in both $\mathrm{CD}^{+}$and $\mathrm{CD} 4^{+} \mathrm{T}$ cells (Figure $2 \mathrm{G}$ and Supplemental Figure 4). Combination treatment also increased TNF- $\alpha$ and granzyme-B (GzB) production by tumor-infiltrating CD8 ${ }^{+} \mathrm{T}$ cells (Figure $2, \mathrm{H}$ and I). Collectively, these data demonstrate that dinaciclib plus anti-PD1 combination treatment augments the number of functionally active $\mathrm{T}$ cells within tumors.

Because dinaciclib can induce tumor cell death, we hypothesized that this in turn could activate local APCs, thereby boosting antitumor responses. Indeed, we found that dinaciclib and antiPD1 combination treatment increased the number of CT26 tumorinfiltrating $\mathrm{CD} 11 \mathrm{c}^{+} \mathrm{DCs}$ and that these cells had higher expression of the activation markers MHC class II (MHCII), CD80, and CD86 when compared with cells from the monotherapy groups (Figure 2, E and F). We observed similar DC activation in the MC38 and MB49 tumor models after combination treatment (Supplemental Figure 2, B and D). We also detected increased MHCII and CD80 
A
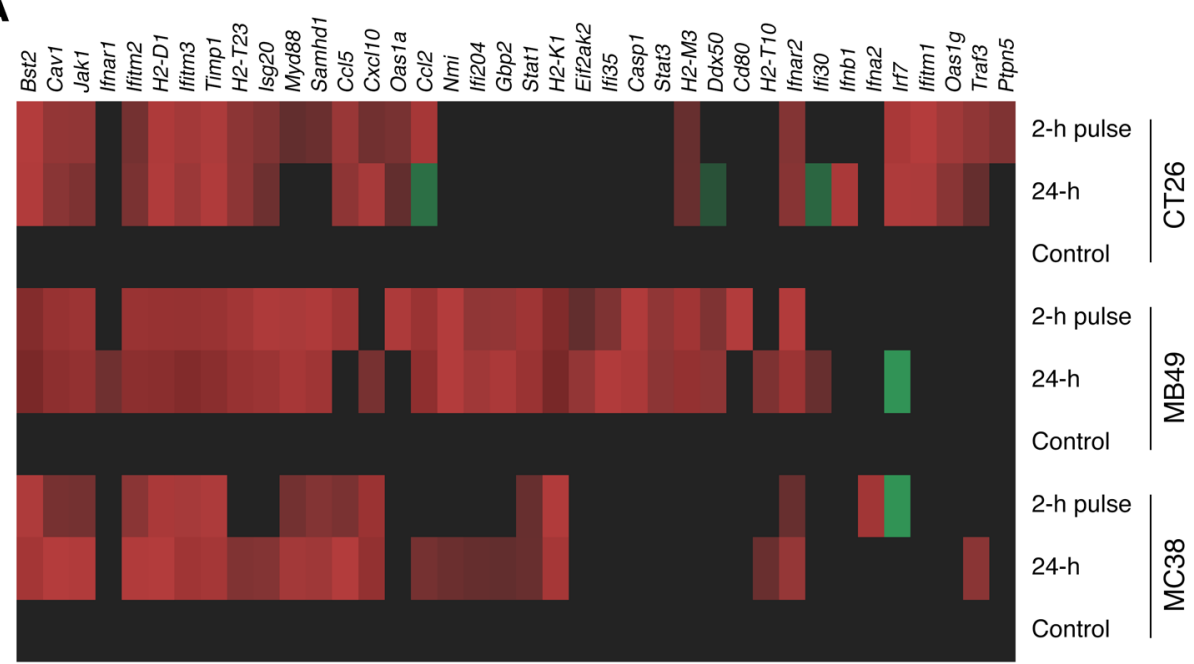

B

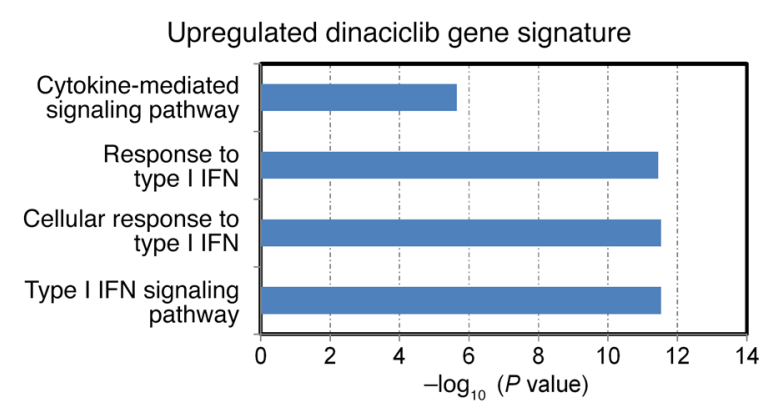

Upregulated anti-PD1 dinaciclib gene signature

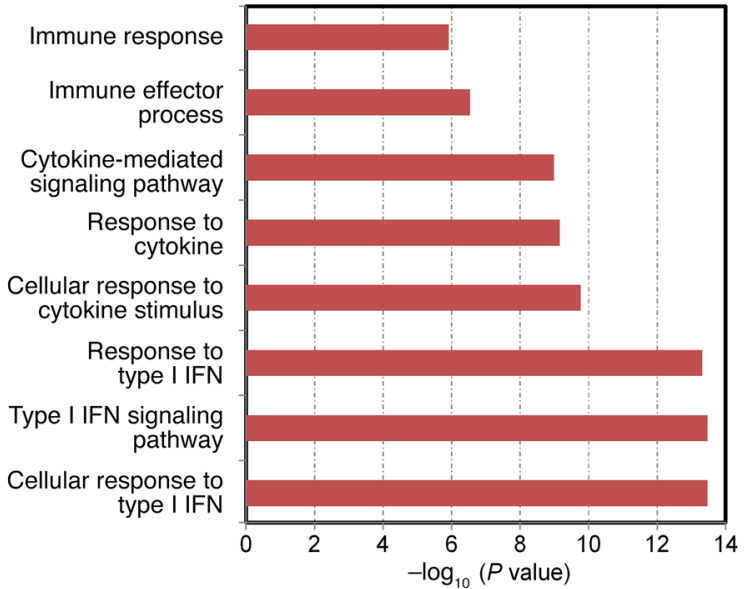

C

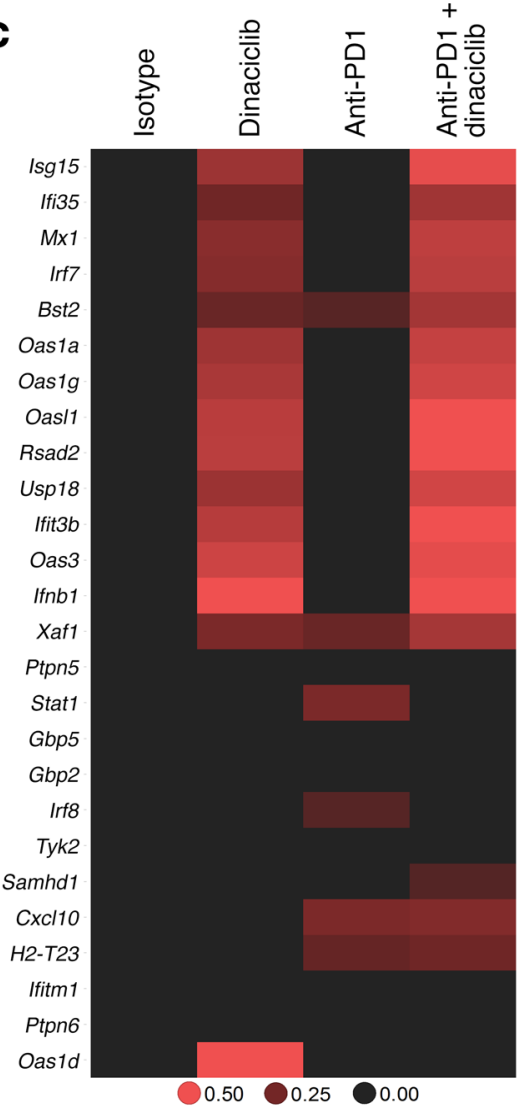

Figure 3. Dinaciclib treatment induces a type I IFN signature within tumors. (A) Fluidigm qPCR analysis of MC38, CT26, and MB49 cells treated with dinaciclib in vitro for 24 hours either continuously or by washing and replacing medium after a 2-hour pulse. Heatmap indicates type I IFN signature genes with a greater-than 2 -fold change $\left(\log _{10}\right.$ scale) over the untreated control and a $P$ value of less than 0.05 . Genes with a FC of less than 2 and a $P$ value of greater than 0.05 are blacked out. (B and C) Mice bearing $100 \mathrm{~mm}^{3}$ MC38 tumors were treated as described in Figure 1. Twenty-four hours after the first dose, tumors were isolated, and gene expression was analyzed by RNA sequencing ( $n=5 /$ group). (B) The top upregulated functional pathways in the dinaciclib and dinaciclib plus anti-PD1 groups as determined by GO analysis and IPA. (C) Expression of type I IFN response genes is depicted by a heatmap showing the $\log _{10} F C$ only of genes that were significantly upregulated $(>2$-fold and $P<0.01$ ) compared with the isotype control group. Genes that were upregulated by less than 2 -fold and that had a $P$ value of greater than 0.01 are blacked out (represented as $F C=0$ ).

expression among $\mathrm{F} 4 / 80^{+}$macrophages (data not shown). These data demonstrate that dinaciclib plus anti-PD1 combination therapy increases both T cell and APC activation and function within the tumor microenvironment compared with either treatment alone.
Dinaciclib induces a type I IFN gene signature in tumor cells. We next examined dinaciclib-induced gene expression by tumor cell lines in vitro using a Fluidigm quantitative PCR (qPCR) array. Interestingly, dinaciclib upregulated type I IFN response genes in 
A
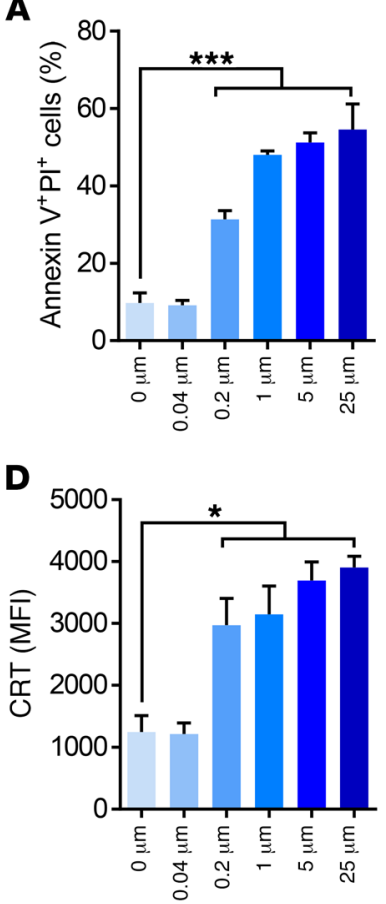

B

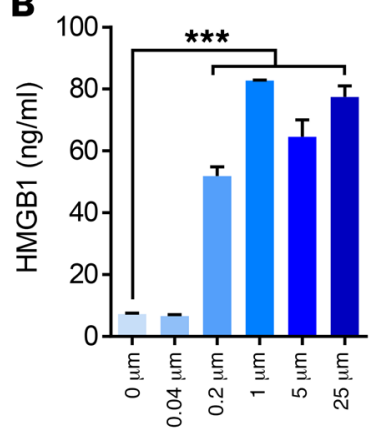

C

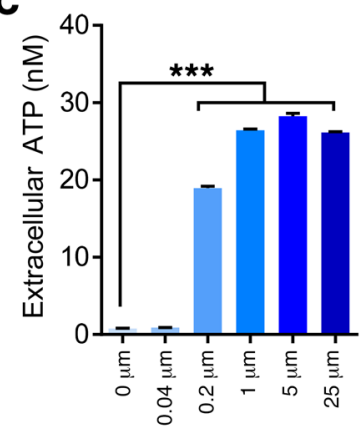

Figure 4. Dinaciclib induces immunogenic cancer cell death. (A-D) CT26 cells were treated for 24 hours in vitro with dinaciclib at the indicated concentrations. Graphical data show (A) the percentage of tumor cell apoptosis, release of (B) HMGB1 and (C) ATP into the culture supernatants, and (D) surface expression of CRT on viable cells. Data represent the mean value \pm SEM of 2 to 3 replicates from 1 representative experiment. ${ }^{*}{ }^{*} P<0.001$ and ${ }^{*} P<0.05$, for comparisons between individual dinaciclib-treated groups and the untreated group $(0 \mu \mathrm{M})$. Statistical data obtained via 1-way ANOVA with Bonferroni post-test. (E) CT26 cells either treated in vitro with dinaciclib or freezethawed were inoculated s.c. into BALB/C mice. After 10 days, mice were rechallenged with live CT26 cells. Shown is the percentage of tumor-free mice pooled from 2 independent experiments. ${ }^{* *} P<0.001$, by log-rank (Mantel-Cox) test.

all 3 syngeneic tumor lines tested. Specifically, dinaciclib induced the gene expression of antiviral molecules (Bst2, Ifnb1, IfitM1, -2, -3, Oas1a, Isg20, and Samhd1), receptor and transcription factors involved in the type I IFN response (Ifnar2, Stat1, Jak1, and Myd88), T cell costimulatory and MHCI molecules (Cav1, H2-T23, and H2D1), and chemoattractant molecules (Ccl5, Cxcl1O, and Ccl2) (Figure $3 \mathrm{~A}$ and Supplemental Table 1). Importantly, the same genes were upregulated when tumor cells were treated for 24 hours or with a 2 -hour pulse of dinaciclib, indicating that dinaciclib induces type I IFN response genes, even when the exposure time was limited to what occurred in vivo (Figure 3A and Supplemental Table 1). We extended this finding in vivo, performing full RNA sequencing on tumors isolated from mice 24 hours after treatment with dinaciclib and anti-PD1 alone or in combination, using the MC38 model, which is the most sensitive to combination therapy. Pathway analysis revealed that the top functional gene categories common for the dinaciclib and combination groups (independent of the anti-PD1 monotherapy group) were related to the cytokine-mediated immune response and the response to type I IFNs (Figure 3B). There were 15 type I IFN-stimulated genes (determined from pathway analysis) that were significantly upregulated (>2-fold; $P<0.01$ ) in the dinaciclib and combination groups (Figure $3 \mathrm{C}$; fold-change in expression over isotype control is shown in Supplemental Table 2). Similar to the in vitro data, the majority of these genes, including Ifnb1, Oas1g, Oas3, Mx1, Oas1a, Irf7, IsG15, Xaf1, and Rsad2, mediate antiviral immunity, cytokine production, and immune cell activation and function $(30,49)$. None of these type I IFN response genes was significantly upregulated in the anti-PD1 monotherapy group $(P>0.01$ vs. the control group, Supplemental Table 2), demonstrating that dinaciclib drives a type I IFN response within the tumor shortly after treatment and independently of anti-PD1 treatment. These data correspond with recent findings showing that type I IFNs are produced by cancer cells within 1 to 4 days of anthracycline-based chemotherapy and mediate immune cell infiltration into the tumor (30). Of note, we did not observe a dinaciclib-induced type I IFN gene signature in tumors on day 4 , when the drug was no longer detectable in the circulation (data not shown).

Dinaciclib induces immunogenic cancer cell death and enhances DC function. The effects of dinaciclib in vivo on both APC activation and type I IFN response suggested that dinaciclib could potentially induce ICD in the tumor. To test this hypothesis, we looked for the hallmarks of ICD in dinaciclib-treated tumor cells in vitro. We observed a dose-dependent induction of apoptosis in tumor cell lines by dinaciclib (Figure 4A and Supplemental Figure 5A) that was associated with increased secretion of HMGB1 and ATP (Figure 4, B and C, and Supplemental Figure 5B) and expression of CRT on the cell surface (Figure 4D and Supplemental Figure 5C). This is similar to what we observed with the known ICD inducer mitoxanthrone (Supplemental Figure 6, A and B). In addition, we detected an increase in cell-surface CRT expression on tumor cells after in vivo treatment with dinaciclib (Supplemental Figure 5D).

As DCs play the key role in the recognition of DAMPs associated with ICD and the subsequent uptake and presentation of tumor antigens, we examined the phagocytosis of dinaciclib-treated tumor cells by DCs. We treated CT26 cells with dinaciclib and then cultured them with mouse bone marrow-derived DCs (BMDCs). We found that dinaciclib-treated tumor cells were efficiently phagocytosed by DCs (Figure 5A and Supplemental Figure 6C), resulting in increased DC maturation, as indicated by the surface expression of MHCII, CD80, and CD86 (Figure 5, B-D). We also found increased secretion of IL-1 $\beta$ in the coculture supernatant (Figure $5 \mathrm{E}$ ). Secretion of IL-1 $\beta$ from DCs in response to purinergic receptor agonists (ATP) and TLR4 ligands (HMGB1) plays an important role in antitumor $\mathrm{T}$ cell priming (33). To test whether antigen presentation was also enhanced, we cultured DCs with dinaciclib-treated, OVA-ex- 
A

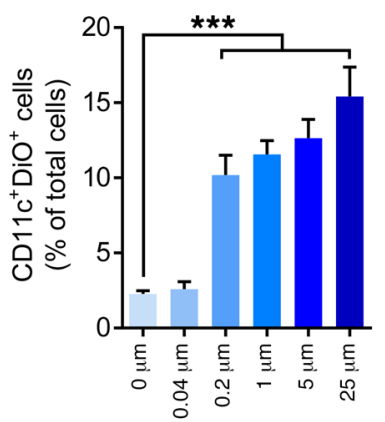

D

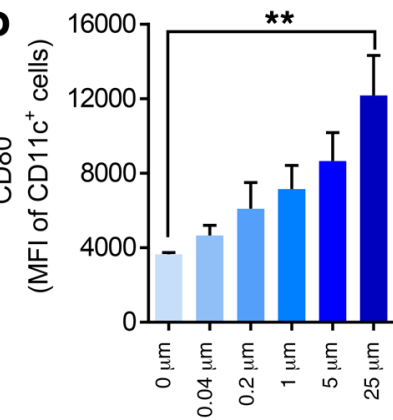

B

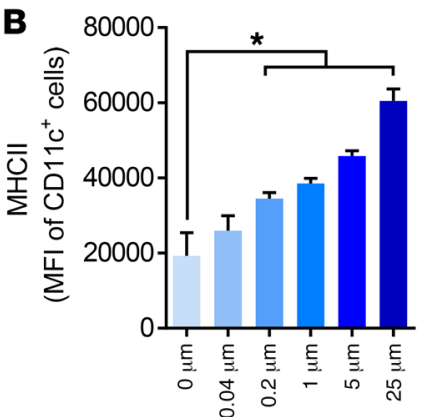

$\mathbf{E}$

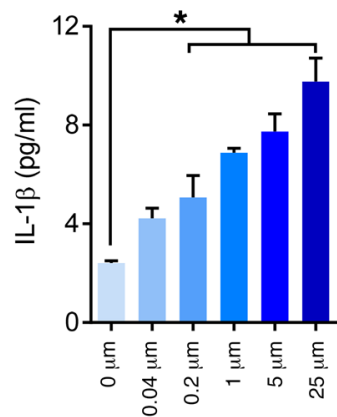

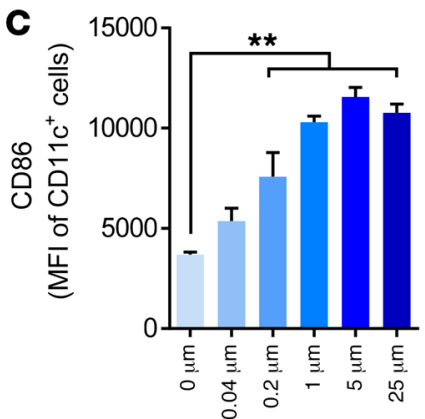

Figure 5. Dinaciclib-treated tumor cells enhance DC function. DiOlabeled CT26 cells were treated with the indicated concentrations of dinaciclib for 24 hours and then cocultured with BMDCs for an additional 24 hours. (A) The percentage of CD11 $C^{+} D C s$ with engulfed tumor cells was assessed by flow cytometry, as was the expression of (B) MHCII, (C) CD86, and (D) CD80 on CD11C ${ }^{+}$DCs after coculture. (E) Secretion of IL-1 $\beta$ into the coculture supernatant was determined by MSD assay. Data represent the mean value \pm SEM of 3 to 4 replicates from 1 representative experiment. ${ }^{* *} P<0.001,{ }^{* *} P<0.01$, and ${ }^{*} P<0.05$, for comparisons between individual dinaciclib dose groups and the untreated group $(0 \mu \mathrm{M})$. Statistical data obtained via 1-way ANOVA with Bonferroni post-test. pressing MC38 tumor cells (MC38-OVA). Along with activation markers, the DCs had increased expression of the OVA peptide SIINFEKL presented by the $\mathrm{H}-2 \mathrm{~Kb}$ MHCI molecule (Supplemental Figure 7). Together, these data clearly show that dinaciclib-killed tumor cells induce DC activation and enhance the processing and presentation of tumor antigens.

Finally, we studied the immunogenic potential of dinaciclib in a vaccination setting. We treated CT26 tumor cells with dinaciclib in vitro and injected them into the left flank of immunocompetent $\mathrm{BALB} / \mathrm{c}$ mice. The mice were then rechallenged with live tumor cells injected into the right flank 10 days later. We observed increased tumor-free survival among mice immunized with dinaciclib-treated dead tumor cells compared with freeze-thawed tumor cells (Figure $4 \mathrm{E}$ ). These results establish dinaciclib as a bona fide ICD inducer.

Increased PD1 expression restrains the antitumor effect of dinac$i c l i b$. Despite ICD induction, the antitumor activity of dinaciclib as a monotherapy was limited, and only in combination with anti-PD1 Ab did dinaciclib enhance tumor suppression (Figure 1, $\mathrm{A}-\mathrm{C}$ ). Recent studies have shown that the induction of PD1 and PD-L1 expression on tumor and associated immune cells can suppress radiation- or chemotherapy-induced immune responses $(50,51)$, and type I IFN signaling plays an important role in mediating PD1 expression on T cells in tumor (52-54). Similarly, we found increased expression of PD1 on tumor-infiltrating $\mathrm{CD}^{+} \mathrm{T}$ cells after in vivo dinaciclib treatment (Figure 6A) and increased expression of PD-L1 on tumor cells after in vitro treatment (Supplemental Figure 8). Thus, to test whether the PD1 signaling axis restrains the antitumor effect of dinaciclib, we compared the effect of dinaciclib monotherapy on the growth of established C57BL/6 MB49 tumors in WT versus PD1-KO mice. As expected, we observed reduced overall tumor growth in PD1-KO mice. Treatment of KO mice with dinaciclib further enhanced TGI, whereas dinaciclib had no significant effect in WT mice (Figure $6 \mathrm{~B})$. These results are in harmony with our initial findings with combined anti-PD1 Ab and dinaciclib treatment and suggest that the downstream antitumor effects of dinaciclib-mediated ICD are limited by expression of PD1 and/or PD-L1.

\section{Discussion}

These results establish the CDK1, $-2,5$, and -9 inhibitor dinaciclib as a bona fide ICD-inducing agent and describe a potential mechanism, whereby combination therapy with anti-PD1 Ab results in enhanced antitumor activity in several murine syngeneic tumor models. In vitro, we found that dinaciclib elicited DAMP expression by tumor cells, which enhanced the phagocytic activity and subsequent processing and presentation of tumor antigens by APCs. Vaccination with dinaciclib-killed tumor cells established a productive immune response and prevented subsequent tumor growth. In vitro and in vivo treatment with dinaciclib stimulated the early expression of type I IFN response genes. Despite these immunogenic properties, dinaciclib had very little effect on tumor growth when dosed as a monotherapy, potentially because of the induction of PD1 expression on T cells or PD-L1 on the tumor. However, when combined with anti-PD1, dinaciclib enhanced $\mathrm{T}$ cell and APC activation within the tumor and significantly improved antitumor efficacy.

Several properties of dinaciclib correspond with its immunogenic property. ER stress is a major feature of ICD and can lead to the expression of immunostimulatory DAMPs, including surface CRT, ATP, and HMGB1 (29). Dinaciclib represses transcription via CDK9 inhibition, and one target of this repression is the shortlived antiapoptotic protein MCL1, which normally protects cells from ER stress and inhibits cell death $(55,56)$. It is interesting to note that anthracyclines, the prototypical ICD-inducing agents, also downregulate MCL1 through transcriptional repression (57). Additionally, dinaciclib has been shown to inhibit cytoprotective components of the IRE1 arm of the unfolded protein response through CDK1 and CDK5 inhibition (58). Through these mecha- 
A

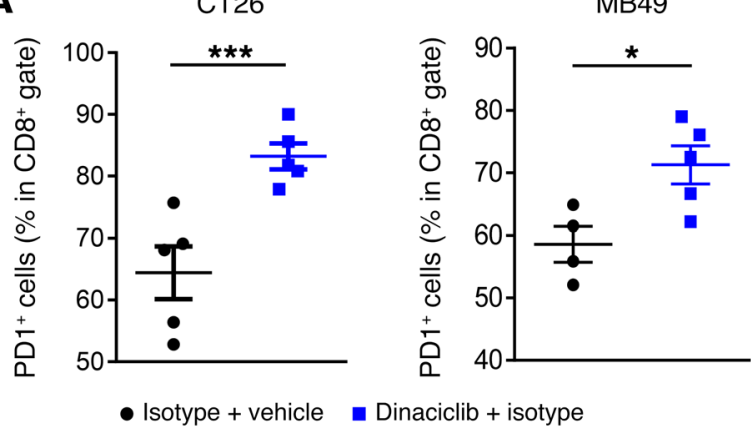

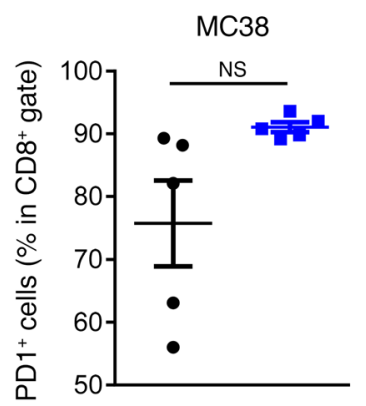

B

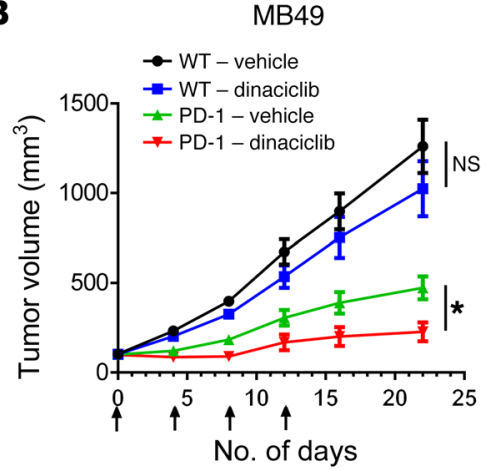

Figure 6. Dinaciclib induces PD1 expression and is efficacious in PD1-KO mice. (A) CT26, MB49, and MC38 tumor-bearing mice were treated as described in Figure 1. Tumors were isolated on day 14 , and PD1 expression on CD8 ${ }^{+}$T cells was analyzed by flow cytometry ( $n=5$ mice per group). (B) WT or PD1-KO mice with established MB49 tumors ( $\left.100 \mathrm{~mm}^{3}\right)$ were treated with dinaciclib or vehicle as described in Figure 1. Tumor growth is represented as the mean tumor volume \pm SEM. Data represent at least 2 independent experiments. Arrows indicate the treatment time points. ${ }^{*} P<0.05$ and ${ }^{* * *} P<0.001$, by unpaired $t$ test (A) applied to calculate 2-tailed $P$ value to estimate statistical difference between vehicle and dinaciclib treatment groups and 2-way ANOVA with Bonferroni post-test (B) applied to assess differences in tumor growth kinetics.

nisms, dinaciclib probably renders target cells more susceptible to ER stress-mediated apoptosis. Accumulation of apoptotic cells, combined with the expression of DAMPs within the tumor, is important for myeloid cell infiltration, activation, tumor antigen presentation, and $\mathrm{T}$ cell priming. Our results are consistent with this scenario, as dinaciclib-induced apoptosis led to increased DC activation and function.

Another key feature of ICD is the elicitation of a type I IFN response. Type I IFN promotes antigen presentation and priming of antitumor T cells, and expression of type I IFN genes has been linked to positive prognosis in response to chemotherapy and radiation $(30,59,60)$. ICD-inducing agents, including anthracyclines, radiation therapy, and oncolytic viruses, have been shown to upregulate type I IFN response genes within the tumor cell (30, $50,60-64)$. Induction of the type I IFN response can be protective in preclinical tumor models, and combination treatment with anti-PD1 has been shown to significantly prolong survival over monotherapy $(65,53)$. Recently, induction of a type I IFN response via radiation therapy was shown to overcome tumor resistance to anti-PD1 (64). However, persistent type I IFN signaling can be immunosuppressive and lead to checkpoint blockade resistance (52). We found that dinaciclib treatment stimulates the transient expression of type I IFN response genes in whole tumors, both when given as a monotherapy or dosed in combination with antiPD1. This pulse of type I IFN gene expression initiated by dinaciclib may be enough to stimulate the immune response without leading to IFN-mediated immunosuppression.

Dinaciclib may additionally regulate tumor and immune cell-intrinsic immunosuppressive mechanisms via modulation of checkpoint inhibitors. Through CDK9 inhibition, dinaciclib has been shown to downregulate the expression of the oncogene $M y c$, and several studies have demonstrated that Myc-driven tumors are especially sensitive to dinaciclib $(43,66,67)$. Recently, Myc inactivation in tumor cells has been linked to downregulation of PD-L1 and CD47, molecules that normally would suppress both adaptive and innate antitumor responses (68). Similarly, CDK5 can phosphorylate Myc, and disruption of CDK5 has been linked to the downregulation of $\mathrm{PD}-\mathrm{L} 1$ expression on medulloblastoma cells, leading to an enhanced immune response in the tumor (69, 70). Conversely, some ICD inducers appear to increase the expression of checkpoint inhibitors and their ligands. The expression of these immunosuppressive molecules, including PD1 and PD-L1, is upregulated on tumor cells or immune cells following radiotherapy, ICD chemotherapy, and type I IFN induction (51, 53, 65, 7173). We found that dinaciclib induced PD-L1 expression on tumor cells in vitro, even after a short exposure to the drug. Additionally, PD1 was induced on tumor-infiltrating $\mathrm{CD}^{+} \mathrm{T}$ cells by dinaciclib monotherapy. This suggests a mechanism whereby dinaciclib treatment leads to PD1- and PD-L1-mediated immunosuppression. Consistent with this hypothesis, dinaciclib monotherapy was more efficacious in PD1-KO mice than in WT mice. Further studies are warranted to more closely examine the regulation by dinaciclib of immunosuppressive mechanisms on both the tumor and the infiltrating immune cells.

It is interesting to consider how dinaciclib exerts proapoptotic effects on tumor cells and yet does not appear to significantly inhibit the generation of an antitumor immune response in vivo. $\mathrm{T}$ cells rely on CDK activity in order to expand, and they are not excluded from the antiproliferative effects of dinaciclib (ref. 74 and our unpublished observations). CDK1 is the major driver of cell-cycle progression, CDK2 has been shown to be essential for optimal $\mathrm{T}$ cell activation and differentiation, and the CDK9 target MCL1 is required for the survival of activated T cells $(75,76)$. Inhibition of CDK2 through $\mathrm{p} 27^{\mathrm{Kip} 1}$, a cell-intrinsic inhibitor of CDKs that is expressed in T cells, has been linked to PD1 and CTLA4 signaling, T cell peripheral tolerance, and CD8 T cell memory, highlighting the importance of CDKs in the maintenance of an active immune response $(75,77-79)$. Yet we observed enhancement of antitumor immunity in our studies and no apparent drop in the number of $\mathrm{T}$ cells. It is possible that dinaciclib may have differential effects on tumor-specific effectors compared with conventional T cells. The CDK2, -5, -7, -9 inhibitor roscovitine has been shown to differentially affect the proliferation of alloreactive versus pathogen-specific and leukemia-specific effector T cells (80, 81). Another possible explanation may lie in the pharmacological properties of dinaciclib itself. In humans, dinaciclib is rapidly elim- 
inated following a 2-hour i.v. infusion, with a terminal half-life of approximately 2 to 3 hours $(41,58,74)$. In mice, the elimination of dinaciclib is even more rapid, with a half-life of less than 1 hour $(48,58)$. In both humans and mice, the direct pharmacodynamic effects of dinaciclib, including inhibition of MCL1, induction of PARP cleavage, and inhibition of PHA-stimulated lymphocyte proliferation, are transient and correlate with plasma drug concentration $(58,74,82)$. In our study, dinaciclib-dependent changes in type I IFN gene expression within the tumor were observed at 24 hours, but not 4 days after dosing (not shown). In vitro, tumor cells were more susceptible to dinaciclib-induced apoptosis than were activated T cells after a 2-hour pulse (Supplemental Figure 9). Thus, a short exposure to dinaciclib may be enough to trigger the immunostimulatory events associated with ICD, without having an overly deleterious effect on the proliferating $\mathrm{T}$ cells.

In light of these findings, it will be interesting to determine whether additional CDK inhibitors are able to promote an antitumor immune response through ICD induction. Of note, a recent study found that the CDK4 and CDK6 inhibitor abemaciclib increases antitumor immunity and enhances the effectiveness of checkpoint blockade, but through a different mechanism than that used by dinaciclib (83). Abemaciclib appears to induce senescence in the tumor cell (rather than apoptosis) and triggers a type III IFN response, which leads to MHCI antigen presentation directly by the tumor cell and PD1 downregulation by CD8 ${ }^{+} \mathrm{T}$ cells (83). Thus, both dinaciclib and abemaciclib enhance the antigenicity of the tumor, but dinaciclib takes a less direct and potentially more-controlled approach via antigen cross-presentation and upregulation of the PD1/PD-L1 axis. Further studies to determine how CDK inhibitors with different selectivities might interact with the immune response would certainly be worthwhile.

Combining ICD-inducing agents and immune checkpoint blockade makes sense intuitively, especially in the context of tumors that lack an existing immune response, and this concept is rapidly gaining traction. It was recently demonstrated that autochthonous tumors in mice, which are nonresponsive to immune checkpoint inhibition, could be sensitized to anti-PD1 and antiCTLA4 by coadministering the ICD-inducing chemotherapeutics oxaliplatin and cyclophosphamide (16). Similarly, checkpoint blockade increases the antitumor response to ICD-inducing radiation therapy $(73,84)$. Several clinical trials that combine checkpoint inhibitors with ICD inducers are ongoing, and the hope is that these combinations will increase the number of patients who can benefit from checkpoint inhibitor therapy, expand the number of indications for treatment with existing chemotherapeutics, and reduce the side effects of chemotherapeutics through dose reduction. Clinical outcomes in these trials will determine whether ICD induction plus immune checkpoint blockade is a valid treatment strategy for cancer. Dinaciclib is currently being tested in combination with the anti-PD1 Ab pembrolizumab in patients with hematological malignancies or advanced breast cancer (ClinicalTrials.gov identifiers NCT02684617 and NCT01676753).

\section{Methods}

Mice and reagents. Six- to eight-week-old female mice were obtained from The Jackson Laboratory (C57BL/6J strain) and Taconic (BALB/ cAnTac and C57BL/6NTac strains). Recombinase-activating gene 1- deficient C57BL/6 ( Rag $\left.^{-/}\right)$mice were originally obtained from the DNAX Research Institute and bred in-house. PD1-KO mice on a C57BL/6NTac background were generated by deletion of exons 2 and 3 from the $P d c d 1$ gene (NCBI gene ID: 18566) via Cas9-mediated gene editing at Taconic Artemis, and the founder animal had perfect end joining at the joining site.

Anti-PD1 (muDX400) was generated by Merck \& Co., Inc., and is a murinized version of a rat anti-mouse PD1 Ab with a mutated D265A mouse IgG1 Fc. The isotype control Ab mouse anti-hexon IgG1 27F11 was generated by Merck \& Co., Inc.. Dinaciclib was generated by Merck \& Co. Inc. and formulated in the vehicle $20 \%$ hydroxypropyl $\beta$ cyclodextrin (Sigma-Aldrich). Mitoxanthrone was obtained from Tocris.

The MC38 cell line (C57BL/6 mouse colon adenocarcinoma) was obtained from the Developmental Therapeutics Program Tumor Repository (Frederick National Laboratory). The MB49 cell line (C57BL/6 mouse urothelial carcinoma) was obtained from Michael O'Donnell (University of Iowa, Iowa City, IA, USA). The CT26 cell line (BALB/c mouse colon adenocarcinoma) was obtained from ATCC. The OVA-expressing MC38 tumor cell line MC38-OVA was generated by Merck \& Co., Inc. Cell lines were cultured in DMEM with 10\% FBS. All cell lines were verified as being free of microbial contamination using an IMPACT I PCR test and genetically authenticated via CellCheck (IDEXX Laboratories).

Detection of apoptosis, surface CRT, and release of ATP and HMGB1. Tumor cell lines were grown to $70 \%$ to $80 \%$ confluence in 6-well plates, washed, and incubated with increasing concentrations of dinaciclib for 24 hours. Dinaciclib-induced tumor cell death was assessed using the Annexin V-Propidium Iodide Apoptosis Detection Kit (eBioscience), and detection of surface CRT is outlined below. Under the same experimental conditions, the collected culture supernatant was assayed for extracellular HMGB1 using an ELISA Kit (Chondrex). Extracellular ATP was quantified using an ENLITEN ATP Assay System Bioluminescence Detection Kit for ATP (Promega), and ATP-derived chemoluminescence was detected on an Analyst HT Multi-Mode Plate Reader (LJL BioSystems).

DC activation, cytokine release, and phagocytosis assays. BM cells were harvested from the femurs of BALB/c mice and cultured in complete RPMI containing mouse recombinant GMCSF ( $50 \mathrm{ng} / \mathrm{ml}$ ) and IL-4 ( $25 \mathrm{ng} / \mathrm{ml}$ ) (Peprotech) for 7 days to generate CD11c ${ }^{+}$DCs. CT26 tumor cells were labeled with DiO (Life Technologies, Thermo Fisher Scientific) and treated with dinaciclib or mitoxantrone for 24 hours. Treated tumor cells were then cocultured with the DCs at a 2:1 ratio for an additional 24 hours. Cell cultures were stained with fluorescence-labeled Abs against CD11c (catalog 117343), MHCII (catalog 107616), CD80 (cata$\log 104731$ ), and CD86 (catalog 105037) (BioLegend) and analyzed by flow cytometry. Tumor cell phagocytosis was detected via analysis of $\mathrm{DiO}$ (tumor)/CD11c (DC) double-positive signal. IL-1 $\beta$ production was detected in coculture supernatants via a V-PLEX MSD Assay (Meso Scale Discovery). Tumor antigen presentation by DCs was assessed using antiH-2Kb-SIINFEKL (clone 25-D1.16; BioLegend).

In vivo experiments. Six- to eight-week-old mice were injected s.c. with MC38 $\left(1 \times 10^{6}\right)$, CT26 $\left(0.3 \times 10^{6}\right)$, or MB49 $\left(0.5 \times 10^{6}\right)$ cells into the lower right flank. Dinaciclib and anti-PD1 treatment commenced when the average tumor size reached $150 \mathrm{~mm}^{3}$ for MC38 and 100 $\mathrm{mm}^{3}$ for CT26 and MB49 tumors. Dinaciclib was administered as 2 i.p. injections of $10 \mathrm{mg} / \mathrm{kg}$, dosed 2 hours apart every 4 days. Anti-PD1 was dosed at $5 \mathrm{mg} / \mathrm{kg}$ every 4 days. For the immunization study, $3 \times 10^{6}$ 
CT26 cells, either freeze-thawed 3 times on dry ice or treated with 1 $\mathrm{mM}$ dinaciclib, were inoculated s.c. into the lower left flank of BALB/c mice. After 10 days, $0.3 \times 10^{6}$ live CT26 cells were inoculated into the right flank, and tumor progression was monitored. Tumor volume was calculated using the formula: $0.5 \times$ length $\times$ width $^{2}$, where the length was the longer dimension. TGI was calculated using the formula: $\left[\left(\mathrm{C}_{\mathrm{t}}-\mathrm{C}_{0}\right)-\left(\mathrm{T}_{\mathrm{t}}-\mathrm{T}_{0}\right)\right] /\left(\mathrm{C}_{\mathrm{t}}-\mathrm{C}_{0}\right) \times 100$, where $\mathrm{C}_{\mathrm{t}}=$ the mean tumor volume of the control group at time $(t) ; \mathrm{C}_{0}=$ the mean tumor volume of the control group at $t_{0} ; \mathrm{T}_{\mathrm{t}}=$ mean tumor volume of the treatment group at $t$; and $\mathrm{T}_{0}=$ mean tumor volume of the treatment group at $t_{0}$.

Ex vivo tumor analysis. For analysis of immune cell populations, isolated tumors were first weighed and then dissociated by gentleMACS (Miltenyi Biotec) and filtered through $70-\mu \mathrm{M}$ cell strainers to generate single-cell suspensions. After counting viable cells, the samples were incubated with Fc $\gamma$ III/IIR-blocking Ab and then stained with fluorochrome-labeled Abs against CD45 (catalog 103128), CD4 (catalog 100546), CD8 (catalog 126610), CD69 (catalog 104530), PD1 (catalog 109110), CD11b (catalog 101242), CD11c (catalog 117343), MHCII (catalog 107616), CD80 (catalog 104731), and CD86 (cata$\log 105037$ ) (BioLegend). Fluorescence data were acquired on a BD LSRFortessa Flow Cytometer and analyzed using FlowJo software. The number of cells within a subset per gram of tumor was calculated using the following formula: (percentage of cells in a subset $\times$ total number of viable cells $) /(100 \times$ tumor weight $)$. For functional analysis, TILs were isolated from dissociated tumors using density-gradient centrifugation and stimulated with PMA and ionomycin in the presence of brefeldin A (BD) for 4 hours. For intracellular staining, cells were fixed, permeabilized, and stained with Abs against IFN- $\gamma$ (cata$\log 554412$ ), TNF- $\alpha$ (catalog 554420), and GzB (catalog 563389) (BD Biosciences). For detection of CRT surface expression by flow cytometry, dinaciclib-treated CT26 cells were stained with fixable viability dye (eBioscience), fixed with $0.25 \%$ paraformaldehyde, stained with anti-CRT Ab (catalog ab2907; Abcam) at 1:100 dilution, and then stained with goat anti-rabbit IgG-AF488 (Life Technologies, Thermo Fisher Scientific), with washing between each step.

For analysis of gene expression, tumors were snap-frozen in liquid nitrogen, and tissues were homogenized into RNA STAT60 (Tel-Test) using a polytron homogenizer, extracting total RNA according to the manufacturer's instructions. After isopropanol precipitation, total RNA was re-extracted with phenol/chloroform/isoamyl alcohol (25:24:1) (Sigma-Aldrich) using phase-lock light tubes (5 Prime; Thermo Fisher Scientific). Libraries for RNA sequencing were prepared by Beijing Genomics Institute (BGI) using $100 \mathrm{ng}$ purified total RNA and the TruSeq Stranded Total RNA RiboZero Library Preparation Kit (Illumina; RS-122-2201), strictly following the manufacturer's instructions (Sample Prep Guide 15031048 E). The resulting library products were quantified with the Agilent 2100 Bioanalyzer and sequenced using an Illumina HiSeq 4000 for a total of $4 \mathrm{~GB}$ of 50-bp paired-end reads per sample. Alignment and transcript quantitation were performed using Omicsoft Array Studio, version 7.2.2.29. Briefly, cleaned reads were aligned to the mouse B38 genome reference using the Omicsoft Aligner, with a maximum of 4 allowed mismatches. Gene level counts were determined by the RSEM algorithm as implemented in Omicsoft Array Studio and using RefGene transcript annotation, prepared March 21, 2014. Upper quartile-normalized fragments per kilobase of transcript per million mapped (UQ-FPKM) were determined and implemented using
Omicsoft Array studio. Gene expression data using UQ-FPKM were $\log _{10}$ transformed. Quality control (QC) was determined by principal component analysis (PCA) and revealed no outliers (data not shown). The average FCs between treatment and isotype treatment groups were generated with an isotype as the vehicle. ANOVA was performed to determine $P$ values. Genes had to have a FC of greater than 2 and a $P$ value of less than 0.01 to be considered significant and qualify for further analysis. Ingenuity Pathway Analysis (IPA) (Ingenuity Systems, QIAGEN Bioinformatics) and Gene Ontology (GO) were used to identify pathways.

For in vitro gene expression analysis, tumor cells were treated with $5 \mu \mathrm{M}$ dinaciclib for 24 hours continuously or by a 2 -hour pulse. After lysis, DNase-treated total RNA was reverse transcribed using QuantiTect Reverse Transcription (QIAGEN) according to the manufacturer's instructions. The primers were obtained commercially from Thermo Fisher Scientific (see the Supplemental Table 1 for the list of primers used). Gene-specific preamplification was done on 10 ng cDNA according to the manufacturer's instructions (Fluidigm). Real-time qPCR was then performed on the Fluidigm Biomark using 2 unlabeled primers at $900 \mathrm{nM}$ each, along with $250 \mathrm{nM}$ FAM-labeled probe (Thermo Fisher Scientific) and TaqMan Universal PCR Master Mix with uracil- $\mathrm{N}$-glycosylase (UNG). Samples and primers were run on a Fluidigm 96.96 Array according to the manufacturer's instructions. Ubiquitin levels were measured in a separate reaction and used to normalize the data by the $\Delta \mathrm{Ct}$ method. Using the mean cycle threshold value for ubiquitin and the gene of interest for each sample, the equation $1.8^{\text {(Ct ubiquitin-Ct gene of interest) }} \times 10^{4}$ was used to obtain the normalized values. The average FC of treated over untreated samples was calculated, and nominal $t$ test analysis was performed to determine $P$ values.

Statistics. One or two-way ANOVA followed by Bonferroni's post test was applied to assess the statistical significance of differences between multiple treatment groups. Data were analyzed using GraphPad Prism 7 (GraphPad Software).

Study approval. All animal procedures were approved by the IACUC of Merck \& Co., Inc., (Kenilworth NJ, USA) in accordance with Association for Assessment and Accreditation of Laboratory Animial Care (AAALAC) guidelines.

\section{Author contributions}

DMSH, AC, EMP, and MH designed the experiments. DMSH, AS, $\mathrm{MC}, \mathrm{CZ}, \mathrm{MS}$, JG, WB, and SJ conducted the research. DMSH, AC, CZ, $\mathrm{SJ}$, and MC analyzed the data. DSMH and AC wrote the manuscript.

\section{Acknowledgments}

We would like to acknowledge the dedication of the members of the analytical cytometry and animal facility at Merck \& Co., Inc. We thank Richard Raubertas (Merck \& Co., Inc., Kenilworth, NJ, USA) for his support with statistical analysis. We thank Pierre Daublain (Merck \& Co., Inc., Kenilworth, NJ, USA) for help with preparing dinaciclib. We acknowledge Rob Kastelein, Terri Mcclanahan, Amy Beebe, Jin-Hwan Han, Danling Gu, Dan Cua, and Rene De Waal Malefyt (Merck \& Co., Inc., Kenilworth, NJ, USA.) for helpful discussions and research support.

Address correspondence to: Alissa Chackerian, Merck \& Co. Inc., 901 S. California Avenue, Palo Alto, California USA. Phone: 650.496.1150; Email: alissa.chackerian@merck.com. 
1. Ansell SM, et al. PD-1 blockade with nivolumab in relapsed or refractory Hodgkin's lymphoma. N Engl J Med. 2015;372(4):311-319.

2. Garon EB, et al. Pembrolizumab for the treatment of non-small-cell lung cancer. $N$ Engl J Med. 2015;372(21):2018-2028.

3. Hamid O, et al. Safety and tumor responses with lambrolizumab (anti-PD-1) in melanoma. $N$ Engl JMed. 2013;369(2):134-144.

4. Robert C, et al. Nivolumab in previously untreated melanoma without BRAF mutation. $N$ Engl $J$ Med. 2015;372(4):320-330.

5. Topalian SL, et al. Safety, activity, and immune correlates of anti-PD-1 antibody in cancer. $N$ Engl JMed. 2012;366(26):2443-2454.

6. Chen L, Han X. Anti-PD-1/PD-L1 therapy of human cancer: past, present, and future. JClin Invest. 2015;125(9):3384-3391.

7. Freeman GJ, et al. Engagement of the PD-1 immunoinhibitory receptor by a novel $\mathrm{B} 7$ family member leads to negative regulation of lymphocyte activation. JExp Med. 2000;192(7):1027-1034 .

8. Keir ME, Butte MJ, Freeman GJ, Sharpe AH. PD-1 and its ligands in tolerance and immunity. Annu Rev Immunol. 2008;26:677-704.

9. Chen L. Co-inhibitory molecules of the B7-CD28 family in the control of T-cell immunity. Nat Rev Immunol. 2004;4(5):336-347.

10. Francisco LM, Sage PT, Sharpe AH. The PD-1 pathway in tolerance and autoimmunity. Immunol Rev. 2010;236:219-242.

11. Mellman I, Coukos G, Dranoff G. Cancer immunotherapy comes of age. Nature. 2011;480(7378):480-489.

12. Sharma P, Allison JP. The future of immune checkpoint therapy. Science. 2015;348(6230):56-61.

13. Topalian SL, Drake CG, Pardoll DM. Immune checkpoint blockade: a common denominator approach to cancer therapy. Cancer Cell. 2015;27(4):450-461.

14. Herbst RS, et al. Predictive correlates of response to the anti-PD-L1 antibody MPDL3280A in cancer patients. Nature. 2014;515(7528):563-567.

15. Hugo W, et al. Genomic and transcriptomic features of response to anti-PD-1 Therapy in metastatic melanoma. Cell. 2016;165(1):35-44.

16. Pfirschke C, et al. Immunogenic Chemotherapy Sensitizes Tumors to Checkpoint Blockade Therapy. Immunity. 2016;44(2):343-354.

17. Rizvi NA, et al. Cancer immunology. Mutational landscape determines sensitivity to PD-1 blockade in non-small cell lung cancer. Science. 2015;348(6230):124-128.

18. Schumacher TN, Schreiber RD. Neoantigens in cancer immunotherapy. Science. 2015;348(6230):69-74.

19. Tumeh PC, et al. PD-1 blockade induces responses by inhibiting adaptive immune resistance. Nature. 2014;515(7528):568-571.

20. Ayers M, et al. IFN- $\gamma$-related mRNA profile predicts clinical response to PD-1 blockade. JClin Invest. 2017;127(8):2930-2940.

21. Bezu L, et al. Combinatorial strategies for the induction of immunogenic cell death. Front Immunol. 2015;6:187.

22. Casares N, et al. Caspase-dependent immunogenicity of doxorubicin-induced tumor cell death. JExp Med. 2005;202(12):1691-1701.
23. Donnelly OG, et al. Measles virus causes immunogenic cell death in human melanoma. Gene Ther. 2013;20(1):7-15.

24. Fucikova J, et al. Human tumor cells killed by anthracyclines induce a tumor-specific immune response. Cancer Res. 2011;71(14):4821-4833.

25. Kroemer G, Galluzzi L, Kepp O, Zitvogel L. Immunogenic cell death in cancer therapy. Annu Rev Immunol. 2013;31:51-72.

26. Korbelik M, Sun J, Cecic I. Photodynamic therapy-induced cell surface expression and release of heat shock proteins: relevance for tumor response. Cancer Res. 2005;65(3):1018-1026.

27. Obeid M, et al. Calreticulin exposure dictates the immunogenicity of cancer cell death. Nat Med. 2007;13(1):54-61.

28. Tesniere A, et al. Immunogenic death of colon cancer cells treated with oxaliplatin. Oncogene. 2010;29(4):482-491.

29. Krysko DV, Garg AD, Kaczmarek A, Krysko O, Agostinis P, Vandenabeele P. Immunogenic cell death and DAMPs in cancer therapy. Nat Rev Cancer. 2012;12(12):860-875.

30. Sistigu A, et al. Cancer cell-autonomous contribution of type I interferon signaling to the efficacy of chemotherapy. Nat Med.2014;20(11):1301-1309.

31. Apetoh L, et al. Toll-like receptor 4-dependent contribution of the immune system to anticancer chemotherapy and radiotherapy. Nat Med. 2007;13(9):1050-1059.

32. DeNardo DG, et al. Leukocyte complexity predicts breast cancer survival and functionally regulates response to chemotherapy. Cancer Discov. 2011;1(1):54-67.

33. Ghiringhelli F, et al. Activation of the NLRP3 inflammasome in dendritic cells induces IL-1beta-dependent adaptive immunity against tumors. Nat Med. 2009;15(10):1170-1178.

34. Halama N, et al. Localization and density of immune cells in the invasive margin of human colorectal cancer liver metastases are prognostic for response to chemotherapy. Cancer Res. 2011;71(17):5670-5677.

35. West NR, Milne K, Truong PT, Macpherson N, Nelson BH, Watson PH. Tumor-infiltrating lymphocytes predict response to anthracycline-based chemotherapy in estrogen receptor-negative breast cancer. Breast Cancer Res. 2011;13(6):R126.

36. Malumbres M, Barbacid M. Cell cycle, CDKs and cancer: a changing paradigm. Nat Rev Cancer. 2009;9(3):153-166.

37. Asghar U, Witkiewicz AK, Turner NC, Knudsen ES. The history and future of targeting cyclin-dependent kinases in cancer therapy. Nat Rev Drug Discov. 2015;14(2):130-146.

38. Baker A, et al. The CDK9 inhibitor dinaciclib exerts potent apoptotic and antitumor effects in preclinical models of MLL-rearranged acute myeloid leukemia. Cancer Res. 2016;76(5):1158-1169.

39. Chen Z, et al. Multiple CDK inhibitor dinaciclib suppresses neuroblastoma growth via inhibiting CDK2 and CDK9 activity. Sci Rep. 2016;6:29090.

40. Desai BM, et al. The anti-melanoma activity of dinaciclib, a cyclin-dependent kinase inhibitor, is dependent on $\mathrm{p} 53$ signaling. PLOS ONE. 2013;8(3):e59588

41. Flynn J, et al. Dinaciclib is a novel cyclin-dependen kinase inhibitor with significant clinical activity in relapsed and refractory chronic lymphocytic leukemia. Leukemia. 2015;29(7):1524-1529.

42. Fu W, et al. The cyclin-dependent kinase inhibitor SCH 727965 (dinacliclib) induces the apoptosis of osteosarcoma cells. Mol Cancer Ther 2011;10(6):1018-1027.

43. Gregory GP, et al. CDK9 inhibition by dinaciclib potently suppresses Mcl-1 to induce durable apoptotic responses in aggressive MYC-driven B-cell lymphoma in vivo. Leukemia. 2015;29(6):1437-1441.

44. Johnson AJ, et al. The novel cyclin-dependent kinase inhibitor dinaciclib (SCH727965) promotes apoptosis and abrogates microenvironmental cytokine protection in chronic lymphocytic leukemia cells. Leukemia. 2012;26(12):2554-2557.

45. Kumar SK, et al. Dinaciclib, a novel CDK inhibitor, demonstrates encouraging single-agent activity in patients with relapsed multiple myeloma. Blood. 2015;125(3):443-448.

46. Varadarajan S, et al. Maritoclax and dinaciclib inhibit MCL-1 activity and induce apoptosis in both a MCL-1-dependent and -independent manner. Oncotarget. 2015;6(14):12668-12681.

47. Wells AD, Morawski PA. New roles for cyclin-dependent kinases in T cell biology: linking cell division and differentiation. Nat Rev Immunol. 2014;14(4):261-270.

48. Parry D, et al. Dinaciclib (SCH 727965), a novel and potent cyclin-dependent kinase inhibitor. Mol Cancer Ther. 2010;9(8):2344-2353.

49. Forster S. Interferon signatures in immune disorders and disease. Immunol Cell Biol. 2012;90(5):520-527.

50. Deng L, et al. STING-dependent cytosolic DNA sensing promotes radiation-induced type I interferon-dependent antitumor immunity in immunogenic tumors. Immunity. 2014;41(5):843-852.

51. Peng J, et al. Chemotherapy induces programmed cell death-ligand 1 overexpression via the nuclear factor- $\mathrm{\kappa} \mathrm{B}$ to foster an immunosuppressive tumor microenvironment in ovarian cancer. Cancer Res. 2015;75(23):5034-5045.

52. Benci JL, et al. Tumor interferon signaling regulates a multigenic resistance Program to immune checkpoint blockade. Cell. 2016;167(6):1540-1554.e12

53. Terawaki S, et al. IFN- $\alpha$ directly promotes programmed cell death- 1 transcription and limits the duration of T cell-mediated immunity. J Immunol.2011;186(5):2772-2779.

54. Gerner MY, Heltemes-Harris LM, Fife BT, Mescher MF. Cutting edge: IL-12 and type I IFN differentially program CD8 $\mathrm{T}$ cells for programmed death 1 re-expression levels and tumor control. J Immunol. 2013;191(3):1011-1015.

55. Zang Y, et al. Carfilzomib and ONX 0912 inhibit cell survival and tumor growth of head and neck cancer and their activities are enhanced by suppression of Mcl-1 or autophagy. Clin Cancer Res. 2012;18(20):5639-5649.

56. Jiang CC, et al. Up-regulation of Mcl-1 is critical for survival of human melanoma cells upon endoplasmic reticulum stress. Cancer Res. 2008;68(16):6708-6717.

57. Wei $\mathrm{G}$, et al. Chemical genomics identifies small-molecule MCL1 repressors and BCL-xL as a predictor of MCL1 dependency. Cancer Cell. 
2012;21(4):547-562.

58. Booher RN, et al. MCL1 and BCL-xL levels in solid tumors are predictive of dinaciclib-induced apoptosis. PLoS ONE. 2014;9(10):e108371.

59. Diamond MS, et al. Type I interferon is selectively required by dendritic cells for immune rejection of tumors. JExp Med. 2011;208(10):1989-2003.

60. Zitvogel L, Galluzzi L, Kepp O, Smyth MJ, Kroemer G. Type I interferons in anticancer immunity. Nat Rev Immunol. 2015;15(7):405-414.

61. Burnette BC, et al. The efficacy of radiotherapy relies upon induction of type $\mathrm{i}$ interferon-dependent innate and adaptive immunity. Cancer Res. 2011;71(7):2488-2496.

62. Melero I, et al. Strict requirement for vector-induced type I interferon in efficacious antitumor responses to virally encoded IL12. Cancer Res. 2015;75(3):497-507.

63. Zamarin D, et al. Localized oncolytic virotherapy overcomes systemic tumor resistance to immune checkpoint blockade immunotherapy. Sci Transl Med. 2014;6(226):226ra32.

64. Wang X, et al. Suppression of type I IFN signaling in tumors mediates resistance to anti-PD-1 treatment that can be overcome by radiotherapy. Cancer Res. 2017;77(4):839-850.

65. Bald T, et al. Immune cell-poor melanomas benefit from PD-1 blockade after targeted type I IFN activation. Cancer Discov. 2014;4(6):674-687.

66. Horiuchi D, et al. MYC pathway activation in triple-negative breast cancer is synthetic lethal with CDK inhibition. JExp Med. 2012;209(4):679-696.

67. Rajput S, Khera N, Guo Z, Hoog J, Li S, Ma CX. Inhibition of cyclin dependent kinase 9 by dinaciclib suppresses cyclin B1 expression and tumor growth in triple negative breast cancer. Oncotarget. 2016;7(35):56864-56875.

68. Casey SC, et al. MYC regulates the antitumor immune response through CD47 and PD-L1. Science. 2016;352(6282):227-231.

69. Dorand RD, et al. Cdk5 disruption attenuates tumor PD-L1 expression and promotes antitumor immunity. Science. 2016;353(6297):399-403.

70. Seo HR, Kim J, Bae S, Soh JW, Lee YS. Cdk5-mediated phosphorylation of c-Myc on Ser-62 is essential in transcriptional activation of cyclin $\mathrm{B} 1$ by cyclin G1. J Biol Chem. 2008;283(23):15601-15610.

71. Deng L, et al. Irradiation and anti-PD-L1 treatment synergistically promote antitumor immunity in mice. J Clin Invest. 2014;124(2):687-695.

72. Ding ZC, et al. Immunosuppressive myeloid cells induced by chemotherapy attenuate antitumor $\mathrm{CD}^{+}$T-cell responses through the PD-1-PD-L1 axis. Cancer Res. 2014;74(13):3441-3453.

73. Dovedi SJ, et al. Acquired resistance to fractionated radiotherapy can be overcome by concurrent PD-L1 blockade. Cancer Res. 2014;74(19):5458-5468.

74. Nemunaitis JJ, et al. A first-in-human, phase 1, dose-escalation study of dinaciclib, a novel cyclin-dependent kinase inhibitor, administered weekly in subjects with advanced malignancies. J Transl Med. 2013;11:259.

75. Chunder N, Wang L, Chen C, Hancock WW, Wells AD. Cyclin-dependent kinase 2 controls peripheral immune tolerance. JImmunol. 2012;189(12):5659-5666.

76. Dzhagalov I, Dunkle A, He YW. The anti-apoptotic Bcl-2 family member Mcl-1 promotes T lymphocyte survival at multiple stages. J Immunol. 2008;181(1):521-528.
77. Jatzek A, Marie Tejera M, Plisch EH, Fero ML, Suresh M. T-cell intrinsic and extrinsic mechanisms of p27Kip1 in the regulation of CD8 T-cell memory. Immunol Cell Biol. 2013;91(2):120-129.

78. Patsoukis N, Brown J, Petkova V, Liu F, Li L, Boussiotis VA. Selective effects of PD-1 on Akt and Ras pathways regulate molecular components of the cell cycle and inhibit T cell proliferation. Sci Signal. 2012;5(230):ra46.

79. Rowell EA, Wang L, Hancock WW, Wells AD. The cyclin-dependent kinase inhibitor p27kip1 is required for transplantation tolerance induced by costimulatory blockade. J Immunol. 2006;177(8):5169-5176.

80. Li L, Wang H, Kim Js, Pihan G, Boussiotis V. The cyclin dependent kinase inhibitor (R)-roscovitine prevents alloreactive $\mathrm{T}$ cell clonal expansion and protects against acute GvHD. Cell Cycle. 2009;8(11):1794-1802.

81. Nellore A, Liu B, Patsoukis N, Boussiotis VA, Li L. The cyclin dependent kinase inhibitor (R)-roscovitine mediates selective suppression of alloreactive human $\mathrm{T}$ cells but preserves pathogen-specific and leukemia-specific effectors. Clin Immunol. 2014;152(1-2):48-57.

82. Gojo I, et al. Clinical and laboratory studies of the novel cyclin-dependent kinase inhibitor dinaciclib (SCH 727965) in acute leukemias. Cancer Chemother Pharmacol. 2013;72(4):897-908.

83. Goel S, et al. CDK4/6 inhibition triggers anti-tumour immunity. Nature. 2017;548(7668):471-475.

84. Twyman-Saint Victor C, et al. Radiation and dual checkpoint blockade activate non-redundant immune mechanisms in cancer. Nature. 2015;520(7547):373-377. 